\title{
Continuous-flow hydration-condensation reaction: Synthesis of $\alpha, \beta$-unsaturated ketones from alkynes and aldehydes by using a heterogeneous solid acid catalyst
}

\author{
Magnus Rueping*, Teerawut Bootwicha, Hannah Baars and Erli Sugiono
}

\section{Full Research Paper}

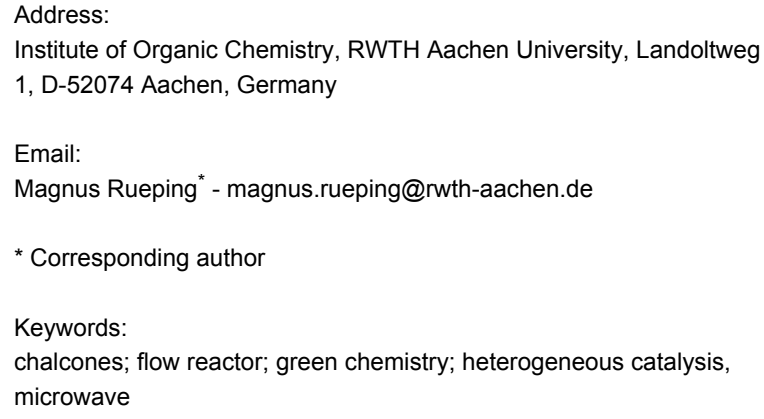

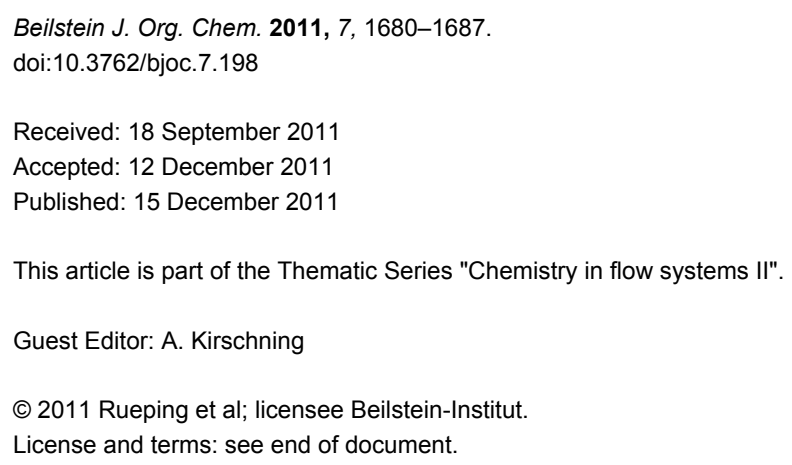

\begin{abstract}
A simple, practical and efficient continuous-flow hydration-condensation protocol was developed for the synthesis of $\alpha, \beta$-unsaturated ketones starting from alkynes and aldehydes by employing a heterogeneous catalyst in a flow microwave. The procedure presents a straightforward and convenient access to valuable differently substituted chalcones and can be applied on multigram scale.
\end{abstract}

\section{Introduction}

In recent years, the development of continuous-flow technologies has expanded considerably and has had a significant impact on modern organic synthetic chemistry. Continuousflow processes offer advantages, such as operational simplicity, energy savings, reduced reagent consumption, and improved mixing quality as well as precise control of the reaction parameters, including pressure, temperature, residence time and heat transfer. The improved operational safety over classical batch reactions reduces the problems of working with hazardous chemicals [1-22]. Furthermore, continuous-flow technologies allow chemical processes to be easily and rapidly scaled up, either by changing the volume of the single reactor, by performing the reaction for an extended reaction time, or by running the reaction in multiple reactors in parallel. Moreover, the products may be collected continuously and formation of byproducts may be reduced by the immediate separation of the products from the reaction mixtures [23-34]. More recently, it has been shown that even asymmetric reactions can be conducted in a continuous-flow fashion [35-40]. Recently, the combination of flow processes and microwave technology has become an interesting endeavour in both academia and in industry. The combination of continuous-flow technology and 
microwave heating offers advantages such as a cleaner reaction profile, reduction of reaction times, higher yields and better selectivity [41-54].

\section{Results and Discussion}

$\alpha, \beta$-Unsaturated ketones are a common motif found in the principal core of a large number of important biologically active compounds. They show pharmacological properties such as antimalarial, antitumor, antiviral, and anti-inflammatory activities [55-60]. They are also well known to be key intermediates in the synthesis of flavones, flavonoids, isoflavonoids and other heterocyclic compounds [61-65]. Consequently, the development of an efficient synthesis to obtain these valuable compounds attracted our interest.

Thus, we decided to develop an efficient continuous-flow synthesis of $\alpha, \beta$-unsaturated ketones starting from alkynes and aldehydes by employing a heterogeneous solid acid catalyst [66-84].

The continuous-flow apparatus for the experiment was set up according to Scheme 1. A $10 \mathrm{~mL}$ reaction vessel was charged with the heterogeneous solid acid catalyst (10 g) and inserted into the reactor. By means of a peristaltic pump the reagents were continuously pumped through the reaction vessel under microwave irradiation $(50 \mathrm{~W})$. The product solution was collected from the outlet tube, which was connected to a $250 \mathrm{psi}$ backpressure regulator of the commercial flow microwave system.

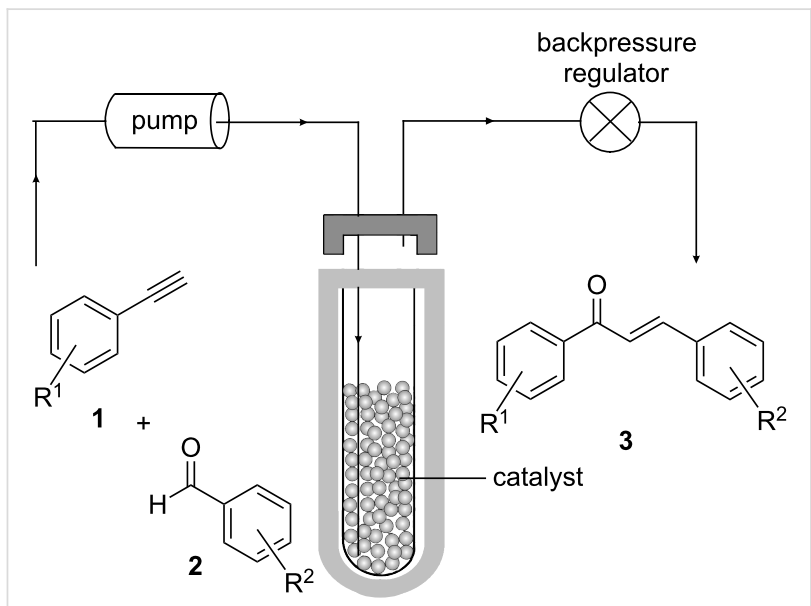

Scheme 1: The schematic arrangement of the continuous-flow system.

Our initial reaction development was focussed on finding the optimal conditions for the continuous-flow reaction of phenylacetylene (1a) with benzaldehyde (2a) applying the ionexchange resin amberlyst-15 [85] as heterogeneous solid acid catalyst. The effects of the substrate concentration, the reaction temperature and the flow rate are summarized in Table 1. Performing the reaction at $80{ }^{\circ} \mathrm{C}$ afforded the $\alpha, \beta$-unsaturated ketone in $70 \%$ yield (Table 1 , entry 5 ). Running the reaction at higher temperature $\left(90{ }^{\circ} \mathrm{C}\right)$ showed a noticeable impact on the conversion and the product was isolated in $84 \%$ (Table 1, entry 2). However, a further temperature increase did not result in improved yield (Table 1, entry 1). Next, we set out to probe the influence of the flow rate on the chemical yield. Performing the reaction at $90{ }^{\circ} \mathrm{C}$ and $0.5 \mathrm{~mL} \mathrm{~min}^{-1}$ gave the product in $84 \%$ yield (Table 1, entry 2). A significant drop in chemical yield was observed when the flow rate increased from $0.5 \mathrm{~mL} \mathrm{~min}^{-1}$ to $1.0 \mathrm{~mL} \mathrm{~min}^{-1}$ (Table 1 , entry 2 versus entry 8 ), indicating that the flow rate should be $0.5 \mathrm{~mL} \mathrm{~min}^{-1}$. Further reaction optimization was accomplished by varying the substrate concentration. While the chemical yields remained constant during an increase from $0.2 \mathrm{M}$ to $0.3 \mathrm{M}$ (Table 1 , entries 2 and 7 ), performing the reaction at lower concentration $(0.1 \mathrm{M})$ afforded the product in lower yield (Table 1, entry 6). A slightly better conversion was obtained when the reaction was performed in dry solvent (Table 1, entry 3 ). However, the best result was achieved when the reaction was conducted under solvent-free conditions (Table 1, entry 4).

To probe the influence of microwave heating on this transformation, we examined the same transformation under batch conditions and without microwave irradiation. As shown in Table 1, under classical batch conditions no product formation was observed when the reaction was performed at $35^{\circ} \mathrm{C}$ (Table 1 , entry 9). By increasing the reaction temperature to $80{ }^{\circ} \mathrm{C}$ using an oil bath, $17 \%$ of the product was isolated after 1 hour reaction time (Table 1, entry 10$)$. Higher isolated yield (47\%) was obtained with a longer reaction time $(3 \mathrm{~h})$ (Table 1 , entry 11$)$. Identical transformation under microwave conditions gave $70 \%$ isolated yield after $30 \mathrm{~min}$ reaction time (Table 1, entry 5).

Having established the optimal reaction conditions, we set out to investigate the scope and applicability of the procedure by employing various alkynes $\mathbf{1}$ and a range of substituted aromatic aldehydes 2 [86]. All the reactions were performed neat, except where the aldehydes were solid. In those cases the reactions were performed in dry DCE. The results are summarized in Table 2.

Generally, the reaction mixture of alkyne $\mathbf{1}$ and aldehyde $\mathbf{2}$ was constantly pumped into the flow cell, filled with the solid acid catalyst and solvent, at the flow rate of $0.5 \mathrm{~mL} \mathrm{~min}{ }^{-1}$ under microwave irradiation. This was followed by a washing procedure with $100 \mathrm{~mL}$ of solvent and then the next substrate was introduced. Importantly, the same catalyst was maintained throughout the reactions. 
Table 1: Optimization of hydration-condensation reactions. ${ }^{a}$

\begin{tabular}{|c|c|c|c|c|c|}
\hline Entry & $\begin{array}{l}\text { Flow rate } \\
{\left[\mathrm{mL} \min ^{-1}\right]}\end{array}$ & Conc. 1a [M] & Heat Source & Temp. $\left[{ }^{\circ} \mathrm{C}\right]$ & Yield [\%] $]^{\mathrm{b}}$ \\
\hline 1 & 0.5 & 0.2 & microwave & 100 & 84 \\
\hline 2 & 0.5 & 0.2 & microwave & 90 & 84 \\
\hline 3 & 0.5 & 0.2 & microwave & 90 & $87^{c}$ \\
\hline 4 & 0.5 & - & microwave & 90 & $91^{d}$ \\
\hline 5 & 0.5 & 0.2 & microwave & 80 & 70 \\
\hline 6 & 0.5 & 0.1 & microwave & 90 & 62 \\
\hline 7 & 0.5 & 0.3 & microwave & 90 & 84 \\
\hline 8 & 1.0 & 0.2 & microwave & 90 & 30 \\
\hline $9^{e}$ & - & 0.1 & - & 35 & n.r. \\
\hline $10^{f}$ & - & 0.2 & oil bath & 80 & 17 \\
\hline $11^{g}$ & - & 0.2 & oil bath & 80 & 47 \\
\hline
\end{tabular}

aReaction conditions: Phenylacetylene (1a) (1.0 equiv), $0.2 \mathrm{M}$ in 1,2-dichloroethane (DCE) and benzaldehyde (2a) (4.0 equiv), $50 \mathrm{~W}, 30$ min. ${ }^{\mathrm{b}}$ Isolated

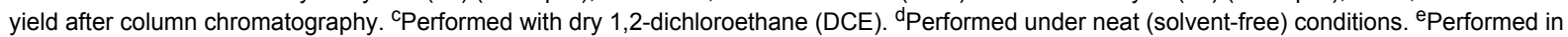
DCM under batch conditions for $24 \mathrm{~h}$. fPerformed in DCE under batch conditions for $1 \mathrm{~h}$. 9Performed in DCE under batch conditions for $3 \mathrm{~h}$.

Table 2: Flow hydration-condensation of alkynes 1 and aldehydes $2 .^{a}$

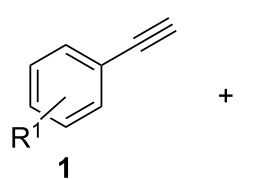<smiles>[R]c1ccc(C=O)cc1</smiles>

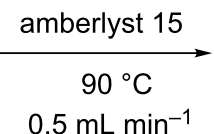<smiles>[R]c1ccc(/C=C/C(=O)c2ccc([R])cc2)cc1</smiles>

\begin{tabular}{|c|c|c|c|c|}
\hline Entry & Alkyne 1 & Aldehyde 2 & Product 3 & Yield $[\%]^{b}$ \\
\hline 1 & $1 a$ & $2 a$ & $3 a$ & $\begin{array}{c}87^{c} \\
91\end{array}$ \\
\hline $2^{c}$ & $1 a$ & $2 b$ & $3 b$ & 96 \\
\hline $3^{c}$ & $1 a$ & 2c & $3 c$ & 98 \\
\hline 4 & $1 a$ & $2 d$ & $3 d$ & 87 \\
\hline
\end{tabular}


Table 2: Flow hydration-condensation of alkynes 1 and aldehydes $2 .^{\text {a }}$ (continued)

5<smiles>C#Cc1ccccc1</smiles>

$1 a$

$6^{c}$<smiles>C#Cc1ccccc1</smiles>

$1 \mathbf{a}$

7<smiles>C#Cc1ccccc1</smiles>

$1 \mathrm{a}$

8<smiles>C#Cc1ccccc1</smiles>

$1 a$

9<smiles>C#Cc1ccccc1</smiles>

$1 \mathrm{a}$

$10^{c}$<smiles>C#Cc1ccccc1</smiles>

1 a

$11^{\mathrm{c}}$<smiles>C#Cc1ccccc1</smiles>

$1 a$

12<smiles>C#Cc1ccccc1</smiles>

$1 \mathrm{a}$

13<smiles>C#Cc1ccc(C)cc1</smiles>

1b

14<smiles>C#Cc1ccc(CC)cc1</smiles>

15

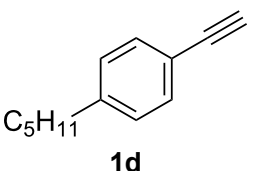<smiles>COc1ccccc1C=O</smiles>

$2 e$<smiles>COc1ccc(C=O)cc1</smiles><smiles>COc1ccc(C=O)c(OC)c1</smiles>

$2 \mathrm{~g}$<smiles>O=Cc1ccc2c(c1)OCO2</smiles>

2h<smiles>O=Cc1ccc(-c2ccccc2)cc1</smiles><smiles>O=Cc1ccccc1Br</smiles>

2j<smiles>O=Cc1ccccc1Cl</smiles>

2k<smiles>O=Cc1cccc(Cl)c1</smiles>

2 I<smiles>O=Cc1ccccc1</smiles>

$2 a$<smiles>O=Cc1ccccc1</smiles>

$2 a$<smiles>O=Cc1ccccc1</smiles>

$2 a$<smiles>COc1ccccc1/C=C/C(=O)c1ccccc1</smiles>

85

$3 e$<smiles>COc1ccc(/C=C/C(=O)c2ccccc2)cc1</smiles>

88

$3 g$<smiles>O=C(/C=C/c1ccc2c(c1)OCO2)c1ccccc1</smiles>

3h<smiles>O=C(/C=C/c1ccc(-c2ccccc2)cc1)c1ccccc1</smiles>

86<smiles>O=C(/C=C/c1ccccc1Br)c1ccccc1</smiles>

$3 \mathbf{j}$<smiles>O=C(/C=C/c1ccccc1Cl)c1ccccc1</smiles>

77

3k<smiles>O=C(/C=C/c1cccc(Cl)c1)c1ccccc1</smiles>

68

3I<smiles>Cc1ccc(C(=O)/C=C/c2ccccc2)cc1</smiles>

98<smiles>CCc1ccc(C(=O)/C=C/c2ccccc2)cc1</smiles>

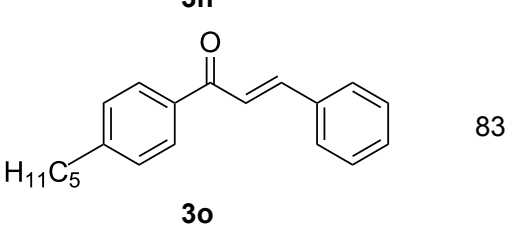


Table 2: Flow hydration-condensation of alkynes 1 and aldehydes $2 .^{\text {a }}$ (continued)

16<smiles>C#Cc1ccc(C(C)(C)C)cc1</smiles>

$1 e$

17<smiles>C#Cc1ccc(OC)cc1</smiles>

18<smiles>C#Cc1ccc(OC)cc1</smiles>

19<smiles>C#Cc1ccc(F)cc1</smiles>

$1 \mathrm{~g}$

20<smiles>C#Cc1ccc(F)cc1</smiles>

$1 \mathrm{~g}$

21

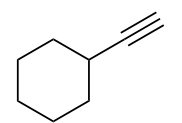

$1 \mathrm{~h}$<smiles>O=Cc1ccccc1</smiles>

$2 a$<smiles>O=Cc1ccccc1</smiles>

$2 a$<smiles>COc1ccc(C=O)cc1</smiles><smiles>O=Cc1ccccc1</smiles>

$2 a$<smiles>O=Cc1ccc2c(c1)OCO2</smiles>

2h<smiles>O=Cc1ccccc1</smiles>

$2 a$<smiles>CC(C)(C)c1ccc(C(=O)/C=C/c2ccccc2)cc1</smiles>

91<smiles>COc1ccc(C(=O)/C=C/c2ccccc2)cc1</smiles><smiles>COc1ccc(/C=C/C(=O)c2ccc(OC)cc2)cc1</smiles>

79

$3 r$<smiles>O=C(/C=C/c1ccccc1)c1ccc(F)cc1</smiles>

3s<smiles>O=C(/C=C/c1ccc2c(c1)OCO2)c1ccc(F)cc1</smiles>
77

$3 t$<smiles>O=C(/C=C/c1ccccc1)C1CCCCC1</smiles>

$3 u$

aReaction conditions: alkynes 1 (1 equiv), aldehydes 2 (4 equiv), solvent-free conditions, $50 \mathrm{~W}$. ${ }^{\mathrm{b}}$ Isolated yield after column chromatography. 'Performed in dry 1,2-dichloroethane (0.2 M).

In general, various aldehydes bearing electron-withdrawing or -donating groups, as well as different substitution patterns were suitable substrates in the reactions. The corresponding $\alpha, \beta$ unsaturated ketones were obtained in good to excellent yields (Table 2, entries 1-12). Applying this procedure no formation of propargylic alcohols was observed and in general no amount of methyl ketone was detected.

Employing the above optimized reaction conditions, further experiments were conducted with a range of substituted alkynes 1 and aldehydes 2 (Table 2, entries 13-21). Again, both electron-rich and electron-poor substrates were well tolerated and the corresponding products were isolated in good yields.

Once the optimal reaction conditions were successfully established on a small scale, we evaluated the potential of this protocol by performing the reactions on a 20 and $49 \mathrm{mmol}$ scale. The reactions of phenylacetylene (1a) with $p$-methyl substituted benzaldehyde $\mathbf{2 b}$ proceeded smoothly providing the corresponding product in excellent isolated yields (Scheme 2).

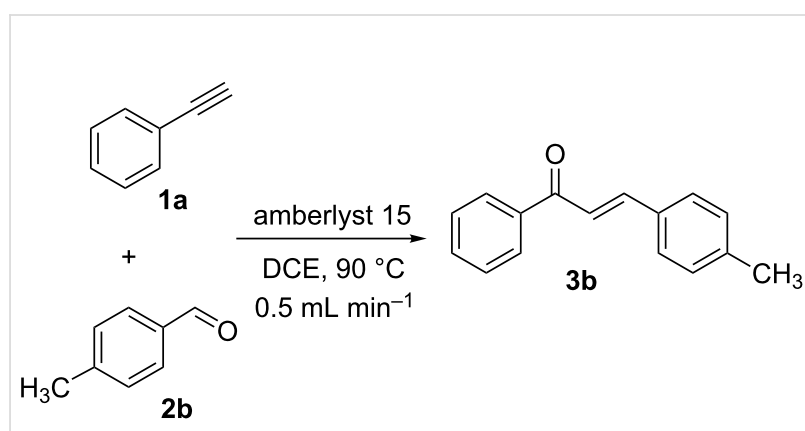

$2.0 \mathrm{~g} \mathrm{(20} \mathrm{mmol)} \mathrm{phenylacetylene} 1 \mathrm{a} ; 4.2 \mathrm{~g} \mathrm{3b}$ (97\% yield) $5.0 \mathrm{~g} \mathrm{(49} \mathrm{mmol)} \mathrm{phenylacetylene} 1 \mathrm{a} ; 9.8 \mathrm{~g} \mathrm{3b}$ ( $91 \%$ yield)

Scheme 2: Preparation of chalcone 3b on larger scale. 


\section{Conclusion}

In conclusion, we have developed a general protocol to access a series of valuable differently substituted chalcones. Starting from commercially available alkynes and aldehydes, a continuous-flow hydration-condensation protocol leads to the desired products in good to excellent yields. The reactions were sequentially introduced into the flow cell and performed several times without the heterogeneous catalyst needing to be changed, demonstrating the high robustness of this catalytic system. Additionally, this new method was readily applied for the preparation of chalcones in multigram quantities. The technology presented is advantageous over classical non-microwave batch reactions in particular with regard to the continuous harvesting of the product, the fast optimization of the reaction parameters, the simple operation and reliability, and the restriction of byproduct formation, especially the formation of methyl ketones and propargylic alcohols.

\section{Experimental}

General procedure for hydration-condensation reaction of phenylacetylene (1a) and benzaldehyde (2a). A solution of phenylacetylene (1a) $(0.4 \mathrm{mmol})$ and benzaldehyde (2a) (1.6 mmol) was pumped through the flow cell filled with amberlyst15 resin (16-50 mesh) (10.0 g) and dry 1,2-dichloroethane at a flow rate of $0.5 \mathrm{~mL} \mathrm{~min}{ }^{-1}$. During this period the reaction vessel in a microwave cavity was irradiated at $90{ }^{\circ} \mathrm{C}(50 \mathrm{~W})$. Following the reaction, $100 \mathrm{~mL}$ solvent was pumped through the flow cell at the same flow rate in order to wash the system, and the combined solutions were evaporated in vacuo. The residue was purified by column chromatography ( $n$-hexane/ DCM $[2: 1$ to $1: 1])$.

\section{Supporting Information}

\section{Supporting Information File 1}

Experimental procedures and characterization of compounds.

[http://www.beilstein-journals.org/bjoc/content/ supplementary/1860-5397-7-198-S1.pdf]

\section{Acknowledgements}

The authors acknowledge the European Research Council for a starting grant.

\section{References}

1. Jas, G.; Kirschning, A. Chem.-Eur. J. 2003, 9, 5708-5723. doi:10.1002/chem.200305212

2. Kikutani, Y.; Kitamori, T. Macromol. Rapid Commun. 2004, 25 , 158-168. doi:10.1002/marc.200300192
3. Kirschning, A.; Solodenko, W.; Mennecke, K. Chem.-Eur. J. 2006, 12, 5972-5990. doi:10.1002/chem.200600236

4. Watts, P.; Haswell, S. J. Chem. Soc. Rev. 2005, 34, 235-246. doi:10.1039/b313866f

5. Geyer, K.; Codée, J. D. C.; Seeberger, P. H. Chem.-Eur. J. 2006, 12, 8434-8442. doi:10.1002/chem.200600596

6. Mason, B. P.; Price, K. E.; Steinbacher, J. L.; Bogdan, A. R.; McQuade, D. T. Chem. Rev. 2007, 107, 2300-2318. doi:10.1021/cr050944c

7. Ahmed-Omer, B.; Brandt, J. C.; Wirth, T. Org. Biomol. Chem. 2007, 5, 733-740. doi:10.1039/b615072a

8. Wiles, C.; Watts, P. Eur. J. Org. Chem. 2008, 1655-1671. doi:10.1002/ejoc.200701041

9. Yoshida, J-i.; Nagaki, A.; Yamada, T. Chem.-Eur. J. 2008, 14, 7450-7459. doi:10.1002/chem.200800582

10. Kirschning, A. Beilstein J. Org. Chem. 2009, 5, No. 15. doi:10.3762/bjoc. 5.15

11. Jähnisch, K.; Hessel, V.; Löwe, H.; Baerns, M. Angew. Chem., Int. Ed. 2004, 43, 406-446. doi:10.1002/anie.200300577

12. Razzaq, T.; Kappe, C. O. Chem.-Asian J. 2010, 5, 1274-1289. doi:10.1002/asia.201000010

13. Wegner, J.; Ceylan, S.; Kirschning, A. Chem. Commun. 2011, 47, 4583-4592. doi:10.1039/c0cc05060a

14. Hartman, R. L.; McMullen, J. P.; Jensen, K. F. Angew. Chem., Int. Ed. 2011, 50, 7502-7519. doi:10.1002/anie.201004637

15. Yamada, Y. M. A.; Torii, K.; Uozumi, Y. Beilstein J. Org. Chem. 2009, 5, No. 18. doi:10.3762/bjoc.5.18

16. Brandt, J. C.; Wirth, T. Beilstein J. Org. Chem. 2009, 5, No. 30. doi: $10.3762 /$ bjoc. 5.30

17. Rolland, J.; Cambeiro, X. C.; Rodríguez-Escrich, C.; Pericàs, M. A Beilstein J. Org. Chem. 2009, 5, No. 56. doi:10.3762/bjoc.5.56

18. Kunz, U.; Turek, T. Beilstein J. Org. Chem. 2009, 5, No. 70. doi:10.3762/bjoc.5.70

19. Gutmann, B.; Glasnov, T. N.; Razzaq, T.; Goessler, W.; Roberge, D. M.; Kappe, C. O. Beilstein J. Org. Chem. 2011, 7, 503-517. doi:10.3762/bjoc.7.59

20. Wiles, C.; Hammond, M. J.; Watts, P. Beilstein J. Org. Chem. 2009, 5, No. 27. doi:10.3762/bjoc.5.27

21. Singh, S.; Köhler, J. M.; Schober, A.; Groß, G. A. Beilstein J. Org. Chem. 2011, 7, 1164-1172. doi:10.3762/bjoc.7.135

22. Fukuyama, T.; Mukai, Y.; Ryu, I. Beilstein J. Org. Chem. 2011, 7, 1288-1293. doi:10.3762/bjoc.7.149

23. Ley, S. V.; Baxendale, I. R. Chimia 2008, 62, 162-168. doi:10.2533/chimia.2008.162

24. Benito-López, F.; Egberink, R. J. M.; Reinhoudt, D. N.; Verboom, W. Tetrahedron 2008, 64, 10023-10040. doi:10.1016/j.tet.2008.07.108

25. Bowman, M. D.; Holcomb, J. L.; Kormos, C. M.; Leadbeater, N. E.; Williams, V. A. Org. Process Res. Dev. 2008, 12, 41-57. doi:10.1021/op700187w

26. Benali, O.; Deal, M.; Farrant, E.; Tapolczay, D.; Wheeler, R. Org. Process Res. Dev. 2008, 12, 1007-1011. doi:10.1021/op700225u

27. Strauss, C. R. Org. Process Res. Dev. 2009, 13, 915-923. doi:10.1021/op900194z

28. Styring, P.; Parracho, A. I. R. Beilstein J. Org. Chem. 2009, 5, No. 29. doi:10.3762/bjoc.5.29

29. Bogdan, A.; McQuade, D. T. Beilstein J. Org. Chem. 2009, 5, No. 17. doi:10.3762/bjoc.5.17

30. Mennecke, K.; Kirschning, A. Beilstein J. Org. Chem. 2009, 5, No. 21. doi:10.3762/bjoc.5.21 
31. Palmieri, A.; Ley, S. V.; Polyzos, A.; Ladlow, M.; Baxendale, I. R. Beilstein J. Org. Chem. 2009, 5, No. 23. doi:10.3762/bjoc.5.23

32. Damm, M.; Glasnov, T. N.; Kappe, C. O. Org. Process Res. Dev. 2010, 14, 215-224. doi:10.1021/op900297e

33. Lehmann, H.; LaVecchia, L. Org. Process Res. Dev. 2010, 14, 650-656. doi:10.1021/op900269y

34. Lv, Y.; Yu, Z.; Su, W. Org. Process Res. Dev. 2011, 15, 471-475. doi:10.1021/op1003083

35. He, P.; Haswell, S. J.; Fletcher, P. D. I.; Kelly, S. M.; Mansfield, A. Beilstein J. Org. Chem. 2011, 7, 1150-1157. doi:10.3762/bjoc.7.133

36. Burguete, M. I.; García-Verdugo, E.; Luis, S. V. Beilstein J. Org. Chem. 2011, 7, 1347-1359. doi:10.3762/bjoc.7.159

37. Schlange, A.; dos Santos, A. R.; Kunz, U.; Turek, T. Beilstein J. Org. Chem. 2011, 7, 1412-1420. doi:10.3762/bjoc.7.165

38. Mak, X. Y.; Laurino, P.; Seeberger, P. H. Beilstein J. Org. Chem. 2009, 5, No. 19. doi:10.3762/bjoc.5.19

39. Cambeiro, X. C.; Martín-Rapún, R.; Miranda, P. O.; Sayalero, S.; Alza, E.; Llanes, P.; Pericàs, M. A. Beilstein J. Org. Chem. 2011, 7, 1486-1493. doi:10.3762/bjoc.7.172

40. Fritzsche, S.; Ohla, S.; Glaser, P.; Giera, D. S.; Sickert, M.; Schneider, C.; Belder, D. Angew. Chem., Int. Ed. 2011, 50, 9467-9470. doi:10.1002/anie.201102331

41. Kappe, C. O.; Dallinger, D.; Murphree, S. Practical Microwave Synthesis for Organic Chemists; Wiley-VCH: Weinheim, 2009.

42. Cablewski, T.; Faux, A. F.; Strauss, C. R. J. Org. Chem. 1994, 59, 3408-3412. doi:10.1021/jo00091a033

43. Bagley, M. C.; Jenkins, R. L.; Lubinu, M. C.; Mason, C.; Wood, R. J. Org. Chem. 2005, 70, 7003-7006. doi:10.1021/jo0510235

44. Baxendale, I. R.; Pitts, M. R. Chim. Oggi 2006, 24, 41-45.

45. Shore, G.; Morin, S.; Organ, M. G. Angew. Chem., Int. Ed. 2006, 45, 2761-2766. doi:10.1002/anie.200503600

46. Baxendale, I. R.; Griffiths-Jones, C. M.; Ley, S. V.; Tranmer, G. K. Chem.-Eur. J. 2006, 12, 4407-4416. doi:10.1002/chem.200501400

47. Glasnov, T. N.; Kappe, C. O. Macromol. Rapid Commun. 2007, 28, 395-410. doi:10.1002/marc.200600665

48. Baxendale, I. R.; Hayward, J. J.; Ley, S. V. Comb. Chem. High Throughput Screening 2007, 10, 802-836.

49. Shore, G.; Tsimerman, M.; Organ, M. G. Beilstein J. Org. Chem. 2009, 5, No. 35. doi:10.3762/bjoc.5.35

50. Shore, G.; Yoo, W.-J.; Li, C.-J.; Organ, M. G. Chem.-Eur. J. 2010, 16, 126-133. doi:10.1002/chem.200902396

51. Dressen, M. H. C. L.; van de Kruijs, B. H. P.; Meuldijk, J.; Vekemans, J. A. J. M.; Hulshof, L. A. Org. Process Res. Dev. 2010, 14, 351-361. doi:10.1021/op900257f

52. Bagley, M. C.; Fusillo, V.; Jenkins, R. L.; Lubinu, M. C.; Mason, C. Org. Biomol. Chem. 2010, 8, 2245-2251. doi:10.1039/b926387j

53. Rueping, M.; Bootwicha, T.; Sugiono, E. Adv. Synth. Catal. 2010, 352, 2961-2965. doi:10.1002/adsc.201000538

54. Wiles, C.; Watts, P. Beilstein J. Org. Chem. 2011, 7, 1360-1371. doi:10.3762/bjoc.7.160

55. Won, S.-J.; Liu, C.-T.; Tsao, L.-T.; Weng, J.-R.; Ko, H.-H.; Wang, J.-P.; Lin, C.-N. Eur. J. Med. Chem. 2005, 40, 103-112. doi:10.1016/j.ejmech.2004.09.006

56. Trivedi, J. C.; Bariwal, J. B.; Upadhyay, K. D.; Naliapara, Y. T.; Joshi, S. K.; Pannecouque, C. C.; De Clercq, E.; Shah, A. K. Tetrahedron Lett. 2007, 48, 8472-8474. doi:10.1016/j.tetlet.2007.09.175

57. Nowakowska, Z. Eur. J. Med. Chem. 2007, 42, 125-137. doi:10.1016/j.ejmech.2006.09.019
58. Aponte, J. C.; Verástegui, M.; Málaga, E.; Zimic, M.; Quiliano, M.; Vaisberg, A. J.; Gilman, R. H.; Hammond, G. B. J. Med. Chem. 2008, 51, 6230-6234. doi:10.1021/jm800812k

59. Nowakowska, Z.; Kędzia, B.; Schroeder, G. Eur. J. Med. Chem. 2008, 43, 707-713. doi:10.1016/j.ejmech.2007.05.006

60. Bhattacharya, A.; Mishra, L. C.; Sharma, M.; Awasthi, S. K.; Bhasin, V. K. Eur. J. Med. Chem. 2009, 44, 3388-3393. doi:10.1016/j.ejmech.2009.02.008

61. Wang, S.; Yu, G.; Lu, J.; Xiao, K.; Hu, Y.; Hu, H. Synthesis 2003, 487-490. doi:10.1055/s-2003-37642

62. Bhat, B. A.; Dhar, K. L.; Puri, S. C.; Saxena, A. K.; Shanmugavel, M.; Qazi, G. N. Bioorg. Med. Chem. Lett. 2005, 15, 3177-3180. doi:10.1016/j.bmcl.2005.03.121

63. Schijlen, E. G. W. M.; de Vos, C. H. R.; Martens, S.; Jonker, H. H.; Rosin, F. M.; Molthoff, J. W.; Tikunov, Y. M.; Angenent, G. C.; van Tunen, A. J.; Bovy, A. G. Plant Physiol. 2007, 144, 1520-1530. doi:10.1104/pp.107.100305

64. Cabrera, M.; Simoens, M.; Falchi, G.; Lavaggi, M. L.; Piro, O. E.; Castellano, E. E.; Vidal, A.; Azqueta, A.; Monge, A.; de Ceráin, A. L.; Sagrera, G.; Seoane, G.; Cerecetto, H.; González, M. Bioorg. Med. Chem. 2007, 15, 3356-3367. doi:10.1016/j.bmc.2007.03.031

65. Siddiqui, Z. N.; Asad, M.; Praveen, S. Med. Chem. Res. 2008, 17, 318-325. doi:10.1007/s00044-007-9067-y

66. Hayashi, A.; Yamaguchi, M.; Hirama, M. Synlett 1995, 195-196. doi:10.1055/s-1995-4909

67. Kokubo, K.; Matsumasa, K.; Miura, M.; Nomura, M. J. Org. Chem. 1997, 62, 4564-4565. doi:10.1021/jo9709458

68. Ishikawa, T.; Mizuta, T.; Hagiwara, K.; Aikawa, T.; Kudo, T.; Saito, S. J. Org. Chem. 2003, 68, 3702-3705. doi:10.1021/jo026592g

69. Curini, M.; Epifano, F.; Maltese, F.; Rosati, O. Synlett 2003, 552-554. doi:10.1055/s-2003-37515

70. Xu, L.-W.; Li, L.; Xia, C.-G.; Zhao, P.-Q. Helv. Chim. Acta 2004, 87, 3080-3084. doi:10.1002/hlca.200490276

71. Viswanathan, G. S.; Li, C.-J. Tetrahedron Lett. 2002, 43, 1613-1615. doi:10.1016/S0040-4039(02)00082-5

72. Park, J. Y.; Ullapu, P. R.; Choo, H.; Lee, J. K.; Min, S.-J.; Pae, A. N.; Kim, Y.; Baek, D.-J.; Cho, Y. S. Eur. J. Org. Chem. 2008, 5461-5469. doi:10.1002/ejoc.200800782

73. Yadav, J. S.; Subba Reddy, B. V.; Vishnumurthy, P. Tetrahedron Lett. 2008, 49, 4498-4500. doi:10.1016/j.tetlet.2008.05.056

74. Jia, H.-P.; Dreyer, D. R.; Bielawski, C. W. Adv. Synth. Catal. 2011, 353, 528-532. doi:10.1002/adsc.201000748

75. Frantz, D. E.; Fässler, R.; Carreira, E. M. J. Am. Chem. Soc. 2000, 122, 1806-1807. doi:10.1021/ja993838z

76. Anand, N. K.; Carreira, E. M. J. Am. Chem. Soc. 2001, 123, 9687-9688. doi:10.1021/ja016378u

77. Gao, G.; Moore, D.; Xie, R.-G.; Pu, L. Org. Lett. 2002, 4, 4143-4146. doi:10.1021/ol026921r

78. Liu, Q.-Z.; Xie, N.-S.; Luo, Z.-B.; Cui, X.; Cun, L.-F.; Gong, L.-Z.; Mi, A.-Q.; Jiang, Y.-Z. J. Org. Chem. 2003, 68, 7921-7924. doi:10.1021/jo034831+

79. Trost, B. M.; Weiss, A. H.; Jacobi von Wangelin, A. J. Am. Chem. Soc. 2006, 128, 8-9. doi:10.1021/ja054871q

80. Scarpi, D.; Galbo, F. L.; Guarna, A. Tetrahedron: Asymmetry 2006, 17, 1409-1414. doi:10.1016/j.tetasy.2006.04.010

81. Ramu, E.; Varala, R.; Sreelatha, N.; Adapa, S. R. Tetrahedron Lett. 2007, 48, 7184-7190. doi:10.1016/j.tetlet.2007.07.196

82. Yang, F.; Xi, P.; Yang, L.; Lan, J.; Xie, R.; You, J. J. Org. Chem. 2007, 72, 5457-5460. doi:10.1021/jo0707535 
83. Krauzy-Dziedzic, K.; Ejfler, J.; Szafert, S.; Sobota, P. Dalton Trans. 2008, 2620-2626. doi:10.1039/b715048b

84. Xu, Z.; Mao, J.; Zhang, Y. Org. Biomol. Chem. 2008, 6, 1288-1292. doi:10.1039/b719624e

85. Amberlyst-15 resin was purchased from Aldrich. Particle size: 16-50 mesh. Matrix: styrene-divinylbenzene (macroreticular).

86. Lower chemical yields were obtained with open-chain aliphatic aldehydes.

\section{License and Terms}

This is an Open Access article under the terms of the Creative Commons Attribution License

(http://creativecommons.org/licenses/by/2.0), which permits unrestricted use, distribution, and reproduction in any medium, provided the original work is properly cited.

The license is subject to the Beilstein Journal of Organic Chemistry terms and conditions:

(http://www.beilstein-journals.org/bjoc)

The definitive version of this article is the electronic one which can be found at: doi:10.3762/bjoc. 7.198 